\title{
OS PARADIGMAS \\ DE INOVAÇÃO E \\ SUAS IMPLICAÇÕES \\ NO DESIGN, NA \\ ENGENHARIA DE \\ SOFTWARE E NO \\ GERENCIAMENTO \\ DE PROJETOS
}

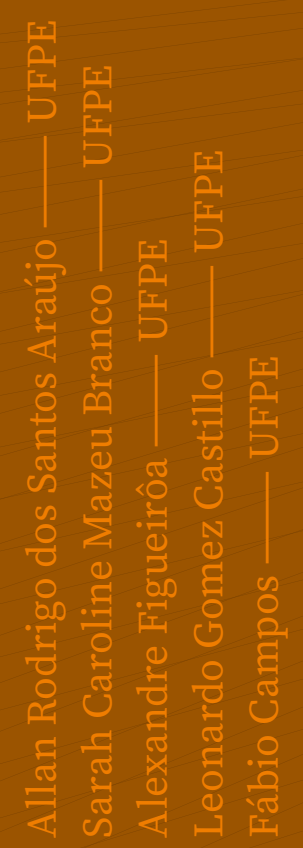




\section{INTRODUÇÃO}

A inovação, um dos temas mais debatidos no contexto da academia e da indústria, tem sido apontada como um dos fatores críticos de sucesso para a sobrevivência das organizações em um mundo globalizado. Porém uma das maiores dificuldades ao estudar inovação tem a ver com o caráter polissêmico da palavra e a falta de consenso a respeito de sua definição. Por exemplo, Tidd (2015) apresenta um compêndio de definições sobre inovação adotadas por diversas organizações e que são encontradas de forma recorrente na literatura conforme apresentado na tabela a seguir: 
DEFINIÇÃO

"A inovação é a boa exploração de novas ideias".
AUTORES

Unidade de Inovação, UK Department of Trade and Industry (2004).

"A inovação industrial abrange as atividades técnicas, comerciais, de projetos, de manufatura e de gestão que estão envolvidas no marketing de um novo (ou aperfeiçoado) produto ou no primeiro uso comercial de um novo (ou aperfeiçoado) processo ou equipamento".

"A inovação não é apenas a comercialização de um grande avanço no estado tecnológico da arte (uma inovação radical), mas até mesmo a utilização de mudanças de pequena escala no know-how tecnológico (uma inovação adicional ou de aperfeiçoamento)".

"A inovação é a ferramenta específica dos empreendedores, pela qual eles se aprofundam nas mudanças como uma oportunidade para negócios ou serviços di-

"As empresas obtêm vantagem competitiva por meio de ações inovadoras. Elas abordam a inovação em seu sentido mais amplo, incluindo tanto novas tecnologias quanto novas maneiras de fazer as coisas".
Chris Freeman, The

Economics of Industrial

Innovation (1982).

Roy Rothwell e Paul Gardiner, Invention, innovation, re-innovation and the role of the user (1985).

Peter Drucker, Innovation and Entrepreneurship (1985). Michael Porter, The Competitive Advantage of Nations (1990).

Dentro desse contexto, compreender os paradigmas de inovação utilizados pelas organizações ao longo do tempo permite sistematizar e compreender as características e o processo evolutivo envolvendo os mesmos. Estudos recentes como Hoppmann, Wu e Johnson (2021) discutem como os paradigmas Direcionado por Tecnologia (Technology Push) e Demandado por Negócios (Market Pull) podem ser combinados por meio de uma estratégia para promover inovação incremental e radical respectivamente. Adicionalmente, buscas em bases de dados como Science Direct, SpringerLink e IEEExplorer mostram uma grande quantidade de estudos recentes a respeito do tema.

Assim, este estudo busca estabelecer o relacionamento entre os paradigmas e disciplinas envolvidas na concepção (projeto) e construção de produtos ou serviços como forma de trazer um senso prático às discussões sobre tais paradigmas. Dessa forma, em particular, a fim de promover uma discussão mais profunda, este estudo escolheu as disciplinas envolvidas no desenvolvimento de artefatos digitais baseados em software, sendo elas: Engenharia de Software, Gerenciamento de Projetos e Design.

Quadro 1. Definições de Inovação. Fonte: Tidd (2015). 


\section{METODOLOGIA}

Este artigo empregou uma abordagem de revisão bibliográfica exploratória com a flexibilidade e liberdade inerentes a esse formato. Contudo, em alguns passos da busca e seleção dos trabalhos, pode-se afirmar que houve o uso de inspiração das atividades previstas por uma Revisão Bibliográfica Sistemática (RBS), conforme descrita por Santos (2018), visando obter melhores e mais consistentes resultados, sem necessariamente estar limitado pela abordagem rigorosa e formal prevista pelo protocolo completo de uma RBS.

Sendo assim, os principais passos foram:

01. Definição de string de busca: Para facilitar encontrar os melhores trabalhos relacionados ao tema por meio da combinação das palavras-chaves a seguir: Paradigmas (paradigms); Inovação (innovation, innovative); e a nomenclatura de alguns dos paradigmas. Dessa maneira, elaborou-se a string: "Innovation" AND "Paradigms" AND ("Technology Push" OR "Market Pull").

02. Busca em bases de dados científicas: Por conveniência e familiaridade dos autores, foram escolhidas as bases Science Direct e Google Scholar.

03. Avaliação preliminar: Por meio do uso de revisão em pares (realizada por dois dos autores) dos artigos em ambas bases de dados e assumindo alguns critérios para seleção dos estudos, tais como:

A. Estudos científicos primários ou secundários;

B. Literatura cinza (livros);

C. Obras em inglês;

D. Trabalhos publicados entre 1990 e 2021;

E. Trabalhos apresentando discussões sobre os temas deste artigo, havendo concordância dos autores.

Com isso, foram selecionados 7 artigos iniciais. 
Além dos 7 (sete) trabalhos classificados como apropriados para este estudo, foi realizada a inclusão manual de 3 (três) outros estudos que não foram identificados na busca (Etapa 2), mas que os autores sabiam atender aos propósitos deste estudo, totalizando 10 (dez) artigos. Por fim, os principais achados foram catalogados para serem discutidos no presente artigo.

\section{RESULTADOS E DISCUSSÃO}

Os resultados obtidos a partir da realização desta pesquisa foram organizados conforme a seguir:

» Paradigmas de Inovação: Descrição e caracterização dos paradigmas de inovação encontrados na literatura;

" Paradigmas e Contribuições: Discussão sobre as sinergias e conflitos existentes entre os paradigmas e as disciplinas de Design, Tecnologia e Gerenciamento de Projetos.

\subsection{PARADIGMAS DE INOVAÇÃO}

Os achados da literatura apontam para a presença de 5 (cinco) paradigmas de inovação distintos e sugerem a coexistência deles ao longo do tempo com uma maior ou menor predominância de acordo com o momento e o contexto, a saber:

》Technology Push (1950-1960);

» Marketing Pull (1960-1970);

》 Coupling of R\&D and Marketing (1970-1980);

"Integrated Business Processes (1980-1990);

》 Systems Integration and Networking (1990-atual).

De maneira geral, os paradigmas coexistem até os dias atuais, sendo a linha do tempo (timeline) apenas uma representação do período do seu surgimento e maior predominância. Discussões específicas são realizadas nas seções apresentadas na Figura 1. 


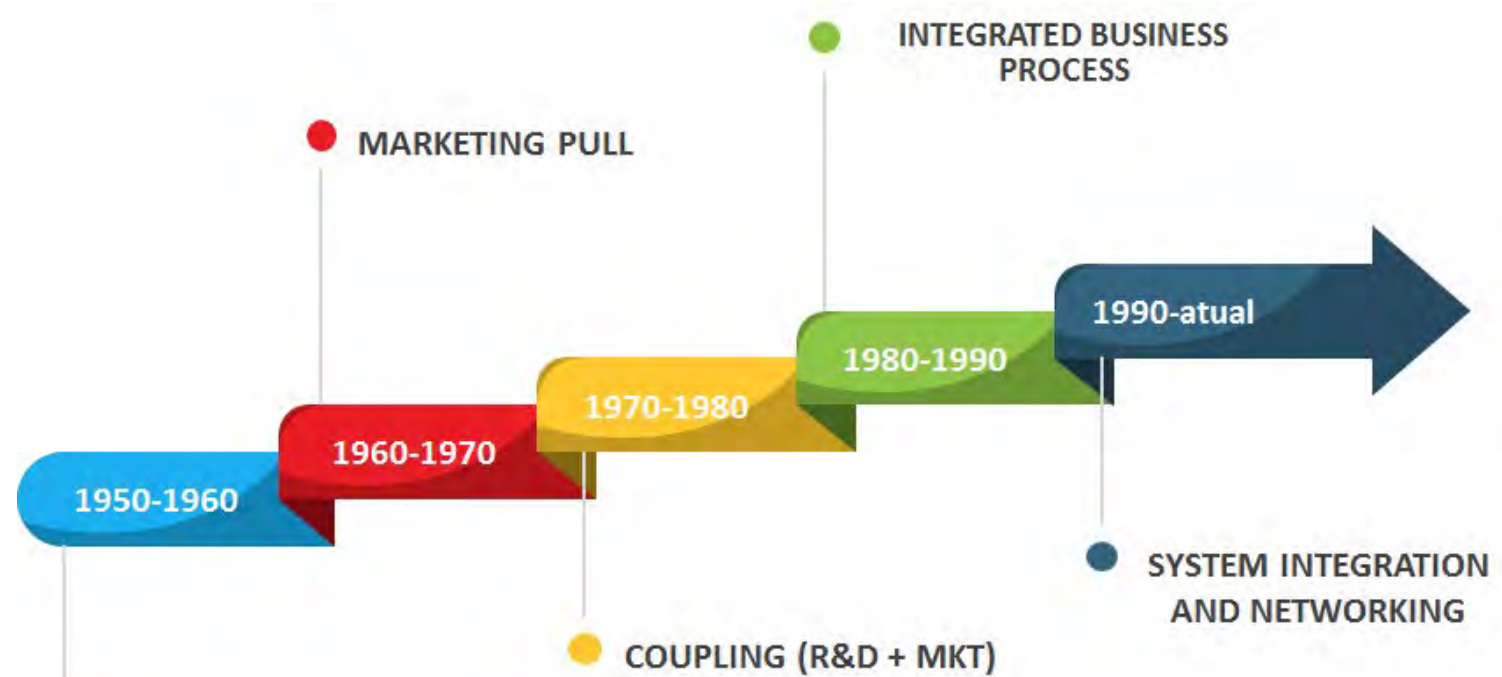

TECHNOLOGY PUSH

\subsubsection{TECHNOLOGY PUSH (DIRECIONADO POR TECNOLOGIA)}

Figura 1 - Linha do

Tempo dos Paradigmas de Inovação. Fonte:

Rothwell (1994); Hobday

(2007); Zizlavsky (2013).
O surgimento desse paradigma de inovação remete ao período após a Segunda Guerra, em que o mercado exibia taxas de crescimento econômico extremamente aceleradas devido a forças como a reconstrução da Europa. Segundo Zizlavsky (2013), surgido entre os anos 1950 e 1960, o paradigma de inovação Technology Push (ou Direcionado por Tecnologia) pode ser descrito como a realização de fases sequenciais, o que por si não representava nenhuma novidade, conforme a Figura 2:

\section{Basic science}

Figura 2 - Fases

do Paradigma

Technology Push. Fonte:

Rothwell (1994).
Neste caso, o processo de inovação era compreendido como uma sequência linear e cronológica de fases (pesquisa, projeto, construção, marketing e vendas). Em particular, o mercado era considerado 
apenas um lugar para que os produtos

pudessem ser destinados sem que houvesse nenhuma influência nas primeiras etapas do processo de inovação.
Figura 3 - Fases

do Paradigma

Marketing Pull. Fonte:

Rothwell (1994).

\subsubsection{MARKETING PULL}

\section{(DEMANDADO POR NEGÓCIOS)}

Mais adiante, já durante os anos 1960 e 1970, a economia demonstrava passar por um período de prosperidade, contudo, graças ao aumento da concorrência e diversificação, tornou-se importante incluir as necessidades do cliente e dos usuários no processo de inovação e, portanto, também no marketing. Do ponto de vista de investimentos, houve uma migração da ciência e tecnologia pura e simples para a criação de novos produtos e suas tecnologias relacionadas de maneira aplicada (ZIZLAVSKY, 2013).

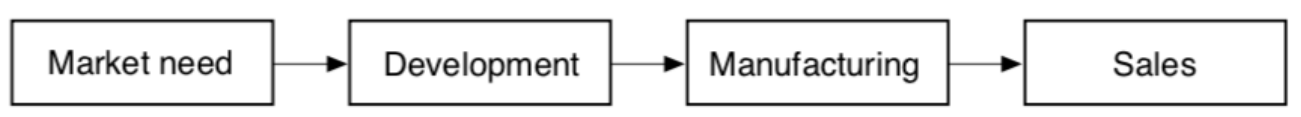

Assim, conforme Rothwell (1994), por conta da necessidade de encontrar um diferencial competitivo em meio a um ambiente marcado por alta competição, surgiu esse novo paradigma que passou a ser orientado a partir de marketing e de negócios - numa clara inversão do paradigma anterior. Nesse caso, apesar da realização sequencial e linear de fases, a criação de novos produtos ocorria a partir da identificação de necessidades de clientes e consumidores através de pesquisas de mercado. 


\subsubsection{COUPLING OF R\&D AND MARKETING} (RELACIONAMENTO ENTRE PESQUISA, DESENVOLVIMENTO E NEGÓCIOS)

Os anos 1970 foram marcados como um período extremamente turbulento do ponto de vista econômico. Em meio a esse ambiente conturbado, as empresas foram forçadas a adotar estratégias de consolidação e racionalização visando à otimização do uso de recursos, aumento do controle e redução do índice de falhas em iniciativas de inovação (ROTHWELL, 1994).

Diferente dos modelos de primeira e segunda geração, o Coupling of $R \& D$ and Marketing (ou Relacionamento entre Pesquisa, Desenvolvimento e Negócios) vincula explicitamente a tomada de decisões das empresas a uma interação entre tecnologia (ciência e tecnologia) e mercado, sendo - por essa razão - também denominado de Interactive Model, ou Modelo Interativo de oportunidades tecnológicas e necessidades de mercado. Nesse paradigma, os loop de feedback são reconhecidos, e, a partir disso, a necessidade das empresas adaptarem novos produtos à concorrência, melhorando a qualidade e os recursos do produto e reduzindo custos (HOBDAY, 2007).

Na definição de Rothwell (1994), trata-se da sequência lógica de um processo, não necessariamente contínuo, dividido em uma série de funcionalidades distintas interagindo em estágios interdependentes (figura 4).

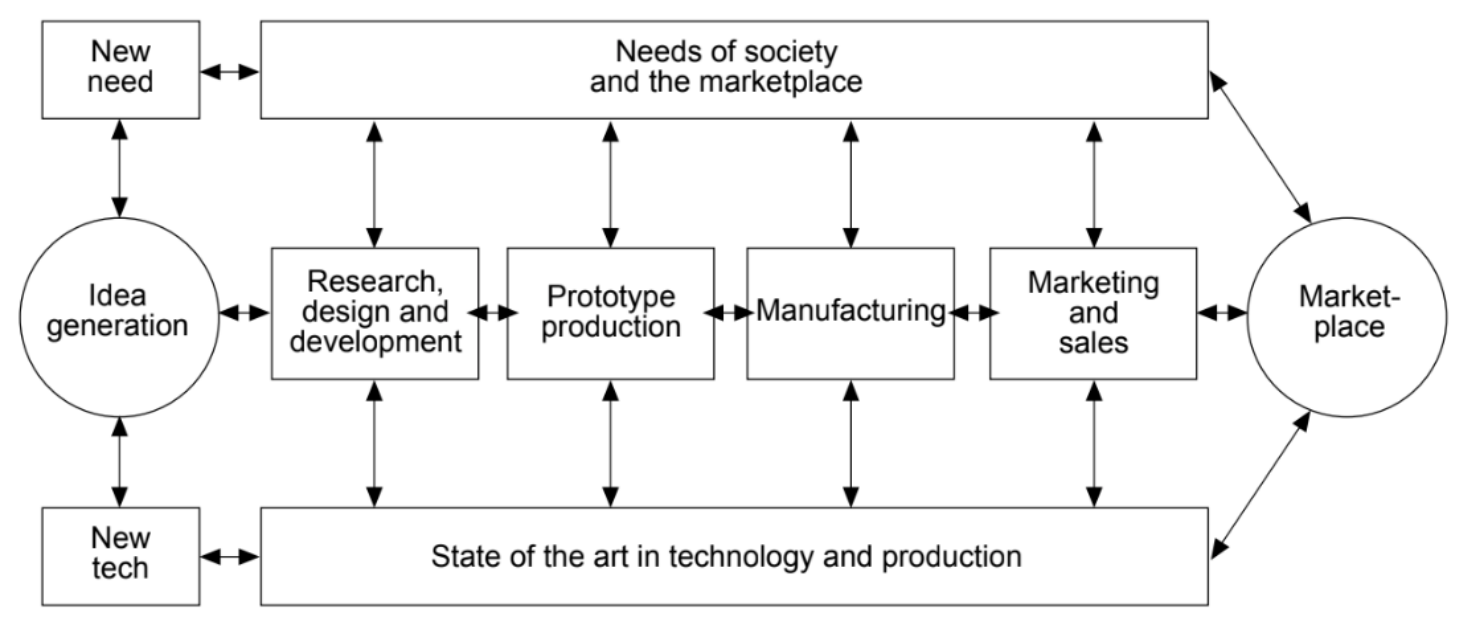

Figura 4 - Coupling of

$R \& D$ and Marketing .

Fonte: Rothwell (1994). 


\subsubsection{INTEGRATED BUSINESS PROCESSES (PROCESSOS DE NEGÓCIOS INTEGRADOS)}

Os anos 1980 (e início dos 1990) marcaram um período de recuperação econômica com as empresas se concentrando - inicialmente - nos seus negócios e tecnologias principais. Nesse contexto, houve um crescimento da importância estratégica do acúmulo de tecnologias. Ao mesmo tempo, a noção de estratégia global (globalização) emergiu, evidenciada pelo aumento do número de alianças estratégicas entre empresas e organizações em geral (ROTHWELL, 1994).

Diante de um cenário global de competição, a redução do tempo de lançamento (time-to-market) de um produto se tornou um forte diferencial competitivo (ROTHWELL, 1994). Devido ao constante encurtamento do ciclo de vida do produto, esse período é caracterizado por uma estratégia baseada no tempo, e, para que isso seja possível, o olhar para fontes externas de ideias e pensamentos torna-se cada vez mais importante (ZIZLAVSKY, 2013).

Dessa forma, um passo decisivo para a consolidação deste modelo de inovação partiu do reconhecimento no ocidente da performance superior das empresas japonesas nos mercados globais (ROTHWELL, 1994). Estas foram as primeiras a identificar sobreposições funcionais significativas entre departamentos e atividades das empresas, caracterizando um alto grau de integração interfuncional, bem como sua integração externa com atividades em outras empresas, incluindo fornecedores, clientes e - em alguns casos - universidades e agências governamentais (HOBDAY, 2007), ganhando destaque conceitos como just-in-time, integração antecipada e o paralelismo.

\subsubsection{SYSTEMS INTEGRATION AND NETWORKING (INTEGRAÇÃO DE SISTEMAS E REDES)}

Em oposição ao período de bonança (rápido crescimento econômico) vivido até meados dos anos 1980, o início dos anos 1990 marcou um período complexo de aumento da concorrência em meio a um ambiente de crescente desempenho, aumento da inflação e aumento das taxas de falência (ROTHWELL, 1994). Assim, este paradigma recebe $o$ nome Systems Integration and Networking (ou Integração de Sistemas e Redes), tendo ampliado o desenvolvimento da quarta geração através do uso da tecnologia da informação, simuladores e sistemas inteligentes (HOBDAY, 2005).

De fato, a mudança dos paradigmas da $4^{\mathrm{a}}$ para a $5^{\mathrm{a}}$ geração representa muito mais uma continuidade (com maior aceleração e intensidade) do que um rompimento. Devido ao acirramento da concorrência (em escala global); redução do time-to-market (ciclo de vida do produto); surgimento de novas tecnologias; expectativas por melhores produtos e serviços; a estratégia baseada nas restrições de tempo se consolidou definitivamente. Embora ser líder em inovação fosse uma característica importante, a capacidade de inovar em um curto espaço de tempo e oferecer novos produtos, serviços ou 
Quadro 2 - Modelos de Gerações de Paradigmas da Inovação. Fonte:

Barbieri e Álvares (2016). processos ao mercado se tornou o principal diferencial competitivo (ZIZLAVSKY, 2013). Como consequência desta nova realidade, houve uma redução do tempo para a realização de pesquisa e desenvolvimento, o que por si só elevou os custos dessas atividades.

\subsubsection{TRABALHOS RELACIONADOS}

Em um dos trabalhos relacionados a este, Barbieri e Álvares (2016) buscam estabelecer um relacionamento entre as diferentes definições e nomenclatura dos paradigmas de inovação encontrados na literatura. Nesse trabalho, os autores abordam diferentes origens para as gerações de paradigmas de inovação, tipos de inovação e modelos de geração de ideias. Em particular, em relação às gerações dos paradigmas, foram identificados os modelos propostos pelo próprio Rothwell (1994). Além dele, Marinova e Phillimore (2003); Tidd (2006); Berkhout, Duin e Ortt (2006); Bochm e Frederick (2010) também criaram suas abordagens para denotar tais paradigmas.

\begin{tabular}{|c|c|c|c|c|c|}
\hline Generation & Rothwell (1994) & $\begin{array}{c}\text { Marinova and } \\
\text { Phillimore (2003) }\end{array}$ & Tidd (2006) & $\begin{array}{l}\text { Berkhout; Duin; } \\
\text { Ortt (2006) }\end{array}$ & $\begin{array}{c}\text { Bochm; Frederick } \\
(2010)\end{array}$ \\
\hline $1^{\text {st }}$ & Technology push & $\begin{array}{l}\text { The black box } \\
\text { model }\end{array}$ & \multirow{2}{*}{$\begin{array}{l}\text { The linear models - } \\
\text { need pull and } \\
\text { technology push }\end{array}$} & Technology push & Technology push \\
\hline $2^{\text {nd }}$ & $\begin{array}{l}\text { Market pull or need } \\
\text { pull }\end{array}$ & $\begin{array}{l}\text { Linear Models } \\
\text { (including } \\
\text { technology push and } \\
\text { need pull) }\end{array}$ & & Market pull & Demand pull \\
\hline $3^{\text {rd }}$ & Coupling model & $\begin{array}{l}\text { Interactive model } \\
\text { (including coupling } \\
\text { and integrated } \\
\text { models) }\end{array}$ & The coupling model & $\begin{array}{l}\text { Combination of } \\
\text { technology push and } \\
\text { market pull }\end{array}$ & $\begin{array}{l}\text { Portfolio } \\
\text { management }\end{array}$ \\
\hline $4^{\text {th }}$ & Integrated model & $\begin{array}{l}\text { Systems model } \\
\text { (including } \\
\text { networking and } \\
\text { national systems of } \\
\text { innovation ) }\end{array}$ & $\begin{array}{l}\text { The parallel lines } \\
\text { model }\end{array}$ & $\begin{array}{l}\text { Cyclic innovation } \\
\text { model }\end{array}$ & $\begin{array}{l}\text { Integrated } \\
\text { management }\end{array}$ \\
\hline $5^{\text {th }}$ & $\begin{array}{l}\text { Parallel and } \\
\text { integrated model }\end{array}$ & Evolutionary model & $\begin{array}{l}\text { Systems integrations } \\
\text { an extensive } \\
\text { networking }\end{array}$ & & Systems integrations \\
\hline $6^{\text {th }}$ & & Innovative milieu & & & $\begin{array}{l}\text { Integration in } \\
\text { network }\end{array}$ \\
\hline
\end{tabular}

Embora haja um relacionamento claro entre os conceitos e nomenclatura, os mecanismos de busca definidos na Seção 2 (Metodologia) indicam que o trabalho de Rothwell (1994) é o mais referenciado e citado entre os achados. 


\subsection{PARADIGMAS E CONTRIBUIÇÕES}

Ao longo deste trabalho, ficou evidente a na-

tureza diversa e plural do fenômeno da inovação seja de produtos, serviços ou processos. Por isso, visando a um maior aprofundamento da discussão e relacionamento com as disciplinas ou ciências que promovem inovação, é necessária uma delimitação do escopo.

Em particular, este estudo se propõe a focar em produtos digitais baseados em software como forma de buscar estabelecer relações entre os paradigmas de inovação e as disciplinas mais recorrentemente envolvidas na criação desse tipo de produto, neste caso: Design, Engenharia de Software (representando a Tecnologia no contexto específico) e Gerenciamento de projetos que historicamente têm dado valiosas contribuições para a inovação desses artefatos. Estes relacionamentos são mais detalhados a seguir:
Quadro 3 - Paradigma

Technology Pull e as

Disciplinas (Engenharia

de Software,

Gerenciamento de

Projetos e Design).

Fonte: Elaborada

pelo autor.

\section{$1^{\mathrm{a}}$ Geração - Technology Pull (1950-1960)}

\begin{tabular}{|c|c|c|}
\hline Engenharia de Software & Gerenciamento de Projetos & Design \\
\hline $\begin{array}{l}\text { As metodologias de engenharia de } \\
\text { software ainda inexistiam, ha- } \\
\text { vendo um foco quase que exclusivo } \\
\text { nas linguagens de programação, } \\
\text { tendo destaque para as linguagens } \\
\text { Assembly, LISP e Fortran (WATT, } \\
\text { 1993) que foram responsáveis } \\
\text { por boa parte dos programas de } \\
\text { computador numa época em que } \\
\text { o hardware estava muito mais em } \\
\text { evidência do que o software. }\end{array}$ & $\begin{array}{l}\text { Ainda dava seus primeiros passos } \\
\text { numa transição de um modelo } \\
\text { artesanal ou improvisado de } \\
\text { gestão para uma administração } \\
\text { das relações humanas. Nessa } \\
\text { época, surgiram técnicas como } \\
\text { o gráfico de Gantt e a WBS (Work } \\
\text { Breakdown Structure - ou Estru- } \\
\text { tura Analítica do Trabalho). Um } \\
\text { exemplo de projeto realizado } \\
\text { utilizando essa abordagem foi o } \\
\text { Projeto Manhattan (KWAK, 2005). }\end{array}$ & $\begin{array}{l}\text { Não foram encontrados regis- } \\
\text { tros sobre o processo de design } \\
\text { empregado nem mesmo sobre a } \\
\text { postura adotada pelo designer. } \\
\text { Contudo, nessa mesma época - de } \\
\text { acordo com Burdek (2010) - a HfG } \\
\text { se desenvolvia como um expoente } \\
\text { do que viria a ser as primeiras } \\
\text { metodologias de design dentro } \\
\text { de um contexto multidisciplinar. }\end{array}$ \\
\hline
\end{tabular}


Quadro 4 - Paradigma

Marketing Push e as

Disciplinas (Engenharia

de Software,

Gerenciamento de

Projetos e Design).

Fonte: Elaborado

pelo autor.
Por sua vez, o advento do paradigma Marketing Push trouxe consigo mudanças nos relacionamentos com as disciplinas de Engenharia de Software, Gerenciamento de Projetos e Design:

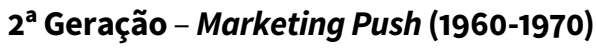

\begin{tabular}{|c|c|c|}
\hline Engenharia de Software & Gerenciamento de Projetos & Design \\
\hline $\begin{array}{l}\text { A Conferência da ONU em Engenha- } \\
\text { ria de Software (1968) se torna um } \\
\text { marco do nascimento da disciplina } \\
\text { ao se discutir abertamente seus } \\
\text { desafios (ex. crise do software), } \\
\text { processos e métodos para sistema- } \\
\text { tização da atividade (WIRTH, 2008). }\end{array}$ & $\begin{array}{l}\text { Aplicação da ciência do geren- } \\
\text { ciamento dentro do contexto de } \\
\text { projetos. Técnicas tradicionais } \\
\text { como o Método do Caminho } \\
\text { Crítico e a Técnica de Avaliação } \\
\text { e Revisão de Programas (PERT) } \\
\text { surgiram (KWAK, 2005). }\end{array}$ & $\begin{array}{l}\text { Disseminação de metodologias de } \\
\text { Design por diversos autores, entre } \\
\text { eles Morris Asimow, Christopher } \\
\text { Alexander e Chris Jones (Burdek, } \\
\text { 2010), com ênfase em sistematização } \\
\text { da atividade de design, faseamento } \\
\text { do processo de design, modula- } \\
\text { rização dos métodos de design. }\end{array}$ \\
\hline
\end{tabular}

Mais adiante, durante os anos 70 e 80, com a introdução do paradigma Coupling of $R \& D$ and Marketing, ou Relacionamento entre Pesquisa, Desenvolvimento e Negócios, houve as seguintes repercussões no relacionamento entre as disciplinas: 
$3^{\mathrm{a}}$ Geração - Coupling of R\&D and Marketing (1970-1980)

\begin{tabular}{|c|c|c|}
\hline Engenharia de Software & Gerenciamento de Projetos & Design \\
\hline $\begin{array}{l}\text { No aspecto das linguagens de } \\
\text { programação, o período associado } \\
\text { ao paradigma ajudou a promover } \\
\text { linguagens estruturas (C, SQL) e } \\
\text { orientadas a objetos (C++, Smalltalk) } \\
\text { que visavam oferecer maior poder } \\
\text { de abstração e produtividade. Do } \\
\text { ponto de vista de modelo de ciclo de } \\
\text { vida, houve um início de transição } \\
\text { do Waterfall (com ciclos de fee- } \\
\text { dback) para o uso incipiente do mo- } \\
\text { delo Espiral (SOMMERVILLE, 2010). }\end{array}$ & $\begin{array}{l}\text { Houve uma intensificação da } \\
\text { aplicação da ciência do gerencia- } \\
\text { mento, consolidando os projetos } \\
\text { como um dos principais meios } \\
\text { para promover a inovação tanto } \\
\text { interna quanto externa nas organi- } \\
\text { zações, com o início da aplicação } \\
\text { da tecnologia da informação. }\end{array}$ & $\begin{array}{l}\text { De acordo com Nijs (2019), as etapas } \\
\text { das metodologias de Design (ex- } \\
\text { ploração do problema, geração e } \\
\text { seleção de alternativas, prototipa- } \\
\text { ção e avaliação) foram mantidas, } \\
\text { porém com uma maior flexibilidade } \\
\text { com relação às iterações necessárias } \\
\text { obedecendo também - ainda que } \\
\text { de maneira tímida - os princípios } \\
\text { do modelo de ciclo de vida em } \\
\text { Espiral (iterativo), além da intro- } \\
\text { dução da orientação do design ao } \\
\text { usuário, fazendo com que o mesmo } \\
\text { começasse a ter um papel fun- } \\
\text { damental dentro do processo de } \\
\text { concepção de produtos e serviços. }\end{array}$ \\
\hline
\end{tabular}

Por sua vez, a partir dos anos 80 e 90, com o surgimento do paradigma de Integrated Business Process, ou Processos de Negócios Integrados, o relacionamento entre as disciplinas evoluiu conforme a seguir:

$4^{\mathrm{a}}$ Geração - Integrated Business Processes (1980-1990)

\section{Engenharia de Software}

Nas linguagens de programação, houve um aumento considerável das opções, marcando um verdadeiro boom na área, destacando-se: Ada, Objective-C, Perl, Haskell, Python, Visual Basic, Java, Ruby, PHP, Javascript, Delphi, etc. (WATT, 1993). Do ponto de vista de processos de desenvolvimento, a abordagem de Big Design Up Front (BAXTER, 2000) ainda dominante e presente (embora não linear) em diversas abordagens em que conceber a solução antes de produzir era fundamental, sendo o Processo Unificado (KRUCHTEN, 2003) o maior expoente desse pensamento.

Gerenciamento de Projetos
Ficou popularizado o PMI (Project
Management Institute) e seu guia de
gerenciamento de projetos denomi-
nado PMBoK (Project Management
Body of Knowledge). Além da publi-
cação do Chaos Report, ou Relatório
do Caos (Standish Group, 1994).

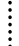

\section{Design}

De acordo com Nijs (2019), em

relação à geração anterior, esta

representou um aumento no nível de envolvimento dos stakeholders e usuários. Com o aumento do poder dos usuários e stakeholders e do papel do designer (mais facilitador do que decisor/diretor), surge o Design Colaborativo, ou Participativo, que envolve uma intensa cocriação durante as etapas do ciclo de vida focadas no projeto de design. Também marca o surgimento do design como pensamento: Design Thinking (Brown, 2008). 
Quadro 5 - Paradigma Relacionamento entre

Pesquisa, Desenvolvimento e Negócios e as

Disciplinas (Engenharia de Software, Gerenciamento

de Projetos e Design). Fonte: Elaborado pelo autor.

Quadro 6 - Paradigma Processos de Negócios

Integrados e as Disciplinas (Engenharia de

Software, Gerenciamento de Projetos e

Design). Fonte: Elaborado pelo autor.

Quadro 7 - Paradigma Integração de Sistemas

e Redes e as Disciplinas (Engenharia de

Software, Gerenciamento de Projetos e

Design). Fonte: Elaboração própria.

Finalmente, desde os anos 90, o paradigma de Systems Integration and Networking, ou Integração de Sistemas e Redes, consolidou o estado da arte atual no relacionamento entre as disciplinas, conforme abaixo:

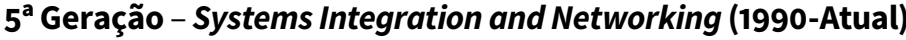

\begin{tabular}{l:lll} 
Engenharia de Software & Gerenciamento de Projetos & Design & \\
\hdashline Considerando os aspectos de & Com o advento das metodologias & A primeina..................................... \\
linguagem de programação, a & ágeis (GRIFFITHS, 2012), final- & Nijs (2019), é o foco deixando de \\
prática ainda se estabelece quase & mente, e dos benefícios do uso & ser valor para se tornar propósito, \\
que totalmente com as tecnolo- & de abordagens como Scrum, Lean & baseado na teoria de complexidade \\
gias criadas cronologicamente & (originário das linhas de produção & sistêmica. Há algumas tentativas, \\
no paradigma anterior. Contudo, & japonesas, Kanban, etc.) a prática & dentre as quais, o Design Emer- \\
do ponto de vista do processo de & tradicional do gerenciamento & gente. Nesse contexto, o modelo \\
engenharia e produção de software, & de projetos mudou dramatica- & se torna completamente coletivo \\
houve uma mudança da aborda- & mente. Primeiramente, o estilo & e o papel do design deixa de ser \\
gem de Big Design Up Front para a & de liderança foi descentralizado, & mediador ou facilitador para ter uma \\
de Little Design Up front (BAXTER, & fazendo com que a hierarquia se & postura mais passiva, em alguns \\
2000), significando que o projeto e & tornasse mais horizontal. Depois, & momentos meramente observacio- \\
sua construção não seriam mais rea- & chegou-se aos times autônomos & nal, não tendo função deliberativa \\
lizados de maneira sequencial, mas & e autogerenciáveis (Squads) dos & em relaçãa às decisões do projeto \\
em paralelo, em ciclos contínuos de & dias atuais, representando o fim do & (solução). Ao mesmo tempo, o \\
feedback, aprendizado e adaptação. & comando e controle como forma & Design hoje é associado à inovação, \\
& de gerir em diversos contextos. & sendo uma ferramenta de gestão \\
& Atualmente, a agilidade é a práxis & e ocupando um lugar estratégico \\
& dentro dos projetos de produtos & nas organizaç̃̃es (MOZOTA, 2010).
\end{tabular}


Atualmente, existem sinergias nas áreas de Design de Interação ( $3^{\text {a }}$ geração) e de Usabilidade com as metodologias ágeis de Gerenciamento de Projetos e de Engenharia de Software. Essa integração foi acelerada a partir do reconhecimento do papel estratégico do Design dentro de uma perspectiva de mudar o pensamento de "construir o projeto corretamente" para "construir o produto/projeto certo". Diversos trabalhos (acadêmicos e do mercado) têm dado contribuições para aumentar essas sinergias, entre eles: Design Thinking (BROWN, 2008), Design Sprint (KNAPP, 2016), Agile UX (BLOKDYK, 2018) e Lean UX (GOTHELF e SEDEN, 2016).
Figura 5 - Relação entre

Paradigmas e Disciplinas

(Produtos Digitais

baseados em Software)

Fonte: Elaborado

pelo autor.

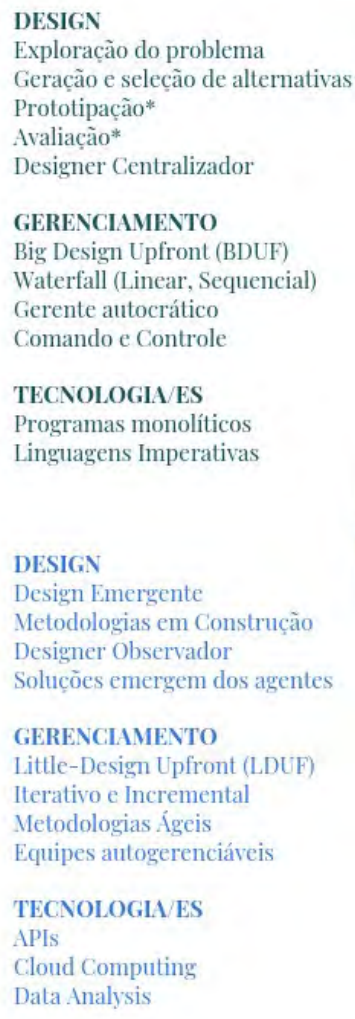

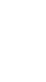

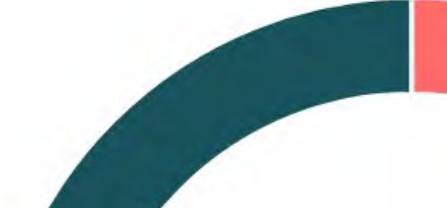

$2^{\mathrm{a}}$ Geração Marketing Pull (1960-1970)
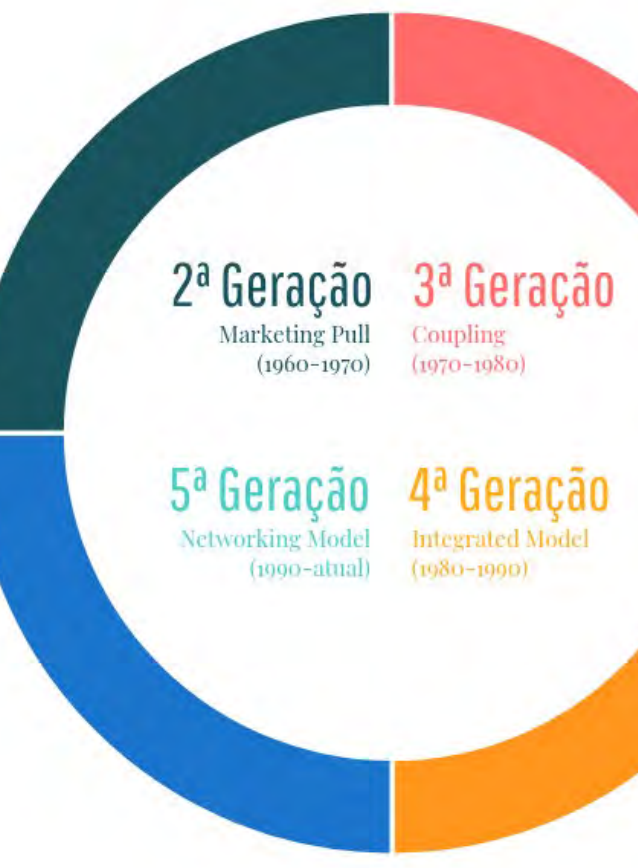

Networking Model (1990-atual)
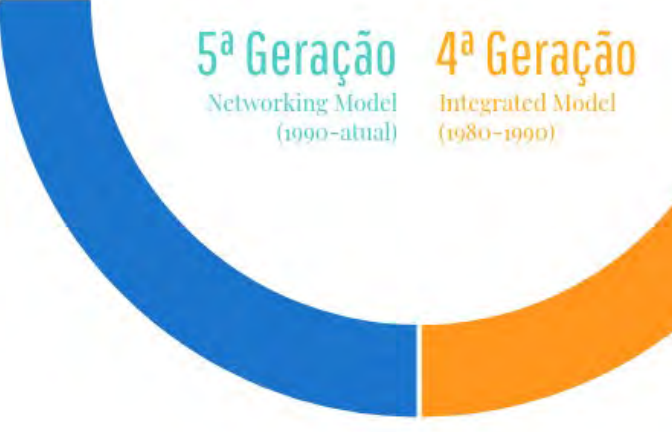

1980-1990)

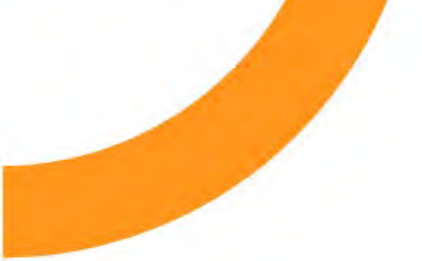

DESIGN

Exploração do problema Geração e seleção de alternativas Prototipação
Avaliacão Design Interativo

GERENCLAMENTO Big-Design Upfront (BDUF) Espiral (Loops/Feedbacks) Gerente Centralizador

Comando e Controle

TECNOLOGIA/ES Programas Estruturados Linguagens Orientadas a Objetos

DESIGN Design centrado no Ustário Design Colaborativo Designer como Facilitador Cocriação

GERENCLIMENTO Big-Design Upfront (BDUF) Iterativo e Incremental Rational Unified Process (RUP)

TECNOLOGIA ES Programas Distribuidos Interfaces Grálicas (GUI) 
Logo, buscar estabelecer estes relacionamentos entre os paradigmas de inovação e suas disciplinas mais importantes para um dado contexto específico é extremamente desafiador, inicialmente, pela própria natureza fluída dos paradigmas que - apesar da definição de Rothwell (1994) e outros autores - não costumam existir em apenas um dado momento do tempo, mas coexistir ao longo do tempo, influenciando os demais.

\section{CONCLUSÃO}

O presente estudo teve por objetivo explorar a literatura existente a respeito dos paradigmas de inovação. Sabe-se que a inovação tem sido uma mola motriz da sociedade promovendo desenvolvimento social e econômico desde os seus primórdios.

Atualmente, na Idade Contemporânea, a inovação tem sido o principal fator de sucesso para que organizações possam sobreviver e vencer em um mercado complexo e globalizado. Por esta razão, o tema inovação (de produtos, serviços e processos) causa interesse tanto na academia, quanto no mercado e na indústria, sendo objeto de iniciativas e estudos multidisciplinares.

Assim, inicialmente, foi proposta uma abordagem que visava realizar uma investigação mais sistemática (numa alusão às revisões e mapeamentos sistemáticos da literatura) para obter melhores resultados. Com isso, chegou-se aos resultados que identificam 10 artigos relacionados à temática estudada por este trabalho, identificando as principais características associadas aos paradigmas de inovação.

Adicionalmente, promoveu-se uma análise - dentro do contexto de artefatos digitais baseados em software e suas disciplinas relacionadas - de modo a mapear as evoluções da Engenharia de Software, do Gerenciamento de Projetos e do Design dentro dos paradigmas de inovação ao longo do tempo. Portanto foram esboçados pilares iniciais para a criação de modelos conceituais a serem criados com base em evidência e estudos empíricos futuramente. 


\section{REFERÊNCIAS}

BARBIERI, J.; ÁLVARES, A.C.T. Sixth generation innovation model: description of a success model. FGV. Revista de Inovação e Administração, 2016.

BAXTER, M. Projetos de Produtos. Guia Prático Para o Design de Novos Produtos. Edição: 2. ed. São Paulo: Blucher, 2000.

BLOKDYK, G. Agile UX. 3. ed. Editora 5STARCooks, 2018.

BROWN, T. Design thinking. Harvard business review, 2008. Disponível em: < https://hbr. org/2008/06/design-thinking>. Acesso em: 11 jul. 2021.

BÜRDEK, Bernhard. Design: História, teoria e prática do design de produtos. São Paulo: Blucher, 2010.

SIGNIFICADO DE INOVAÇÃO: O Que É Inovação. In: DICIONÁRIO de significados. Disponível em: <https://www.significados. com.br/inovacao/> Acesso em: 18 set. 2019. GOTHELF, J.; SEDEN, J. Lean UX. 2. ed. Massachusetts: Editora O`Reilly, 2016

GRIFFITHS, M. PMI-ACP Exam Prep: Rapid Learning to Pass the Pmi Agile Certified Practitioner (Pmi-acp) Exam, 2012.

HOBDAY, M. Firm-level innovation models: perspectives on research in developed and developing countries. Technology analysis \& strategic management, v. 17, n. 2, p. 121-146, 2005. 
HOPPMANN, J.; WU, G.; JOHNSON, J. The impact of demand-pull and technology-push policies on firms' knowledge search. Technological Forecasting \& Social Change Journal, v. 170, 2021.

KNAPP, J. et al. Sprint: How to Solve Big Problems and Test New Ideas in Just Five Days. Nova York: Simon \& Schuster, 2016. KRUCHTEN, P. The Rational Unified Process: An Introduction. 3. ed. Boston: AddisonWesley, 2003.

KWAK, Y. A brief history of project management. In: The story of managing projects. Westport: Quorum Books, 2005.

MOZOTA, B.B. Gestão do Design. 1. ed. Porto Alegre: Editora Bookman, 2010.

NIJS, D. Introduction: towards a new design discipline. in: NIJS, D. (Ed.) Advanced Imagineering: designing innovation as collective creation. Cheltenham: Edward Elgar Publishing, 2019.

ROTHWELL, R. Successful industrial innovation: critical factors for the 1990s. $R \& D$ Management, v. 22, p. 221-240, 1992.

SANTOS, A. Seleção de Método de Pesquisa. Guia para Pós-Graduandos em Design e Áreas Afins. Curitiba: Editora Insight, 2018. . Systems integration and networking: the fifth generation innovation process. (Artigo) CHAIR HYDROQUEBEC CONFERENCE EN GESTION DE AL TECHNOLOGIE, Montreal, Canada, 28 maio 1993.
Towards the Fifth-generation

Innovation Process. International Marketing Review. 1994

SOMMERVILLE, I. Software Engineering. 9.

ed. Harlow: Addison Wesley, 2010

STANDISH GROUP INTERNATIONAL. The Chaos Report. 1994. Technical Report.

TIDD, J.; BESSANT, J. Gestão da Inovação.

Porto Alegre: Bookman, 2015.

WATT, D.; WONG, S. Programming Languages Concepts and Paradigms. New Jersey:

Prentice Hall,1993.

WIRTH, K. A brief history of software engineering. IEEE Annals of the History of Computing v. 30, n. 3, p. 32-39, 2008.

ZIZLAVSKY, O. Past, Present and Future of the Innovation Process. International Journal of Engineering Business, 2013. 